\title{
A scheme for symmetrization verification
}

\author{
Pedro Sancho \\ Centro de Láseres Pulsados, CLPU. \\ E-37008, Salamanca, Spain
}

\begin{abstract}
We propose a scheme for symmetrization verification in two-particle systems, based on one-particle detection and state determination. In contrast to previous proposals, it does not follow a Hong-Ou-Mandel-type approach. Moreover, the technique can be used to generate superposition states of single particles.
\end{abstract}

PACS: 03.65.-w; 03.67.Mn

\section{Introduction}

Non-factorizable states play a fundamental role in quantum theory. There are two classes of non-separable states, entangled and (anti)symmetrized ones. Recently, there has been a boom in the theoretical and experimental study of the first ones, specially concerning their generation, verification and potential applications in the fields of quantum information and conceptual foundations of quantum theory. Here, we are mainly interested into the question of verification. References [1,2] are two good introductions to the problem.

In contrast, there are not so many studies concerning the same issue for (anti)symmetrized states of massive particles. In quantum theory, two identical particles whose wave functions overlap at a given instant are indistinguishable at any subsequent time. If one localizes one of the particles at any instant after the overlapping, it is impossible to identify it with any of the initial ones. In order to correctly describe this impossibility the wave function of the two-particle system cannot be a product state (even for non-interacting particles) but must be symmetrized in the case of bosons and antisymmetrized for fermions.

In quantum optics, the standard test for determining identicalness, i. e., for testing if two photons are really in indistinguishable states (and consequently it is necessary to symmetrize the state) is based on the Hong-Ou-Mandel (HOM) arrangement [3]. This technique is based on the interference of the photons at a beam splitter [4].

Several authors have proposed to use the same approach for massive particles $[5,6,7,8]$. In $[5,6]$, mesoscopic electron beam splitters based on electron beam 
lithography on a GaAs high-mobility two-dimensional electron gas system have been experimentally used to test the antibunching of fermions, an unequivocal demonstration of their antisymmetrization. Several other arrangements can be used as beam splitters for beams of massive particles $[9,10]$, providing also an alternative basis for feasible massive HOM-type tests. Unfortunately, their manipulation is much more delicate than their counterparts for light beams, making more difficult the application of the technique to massive particles.

The importance of having methods able to verify (anti)symmetrization can be easily understood. For instance, in [11] it has been suggested to study massive two-particle interferometry by diffraction gratings. One expects novel effects when identical particles arrive in (anti)symmetrized states. In order to experimentally test such effects we must know in advance if the particles incident on the diffraction grating are in (anti)symmetrized states. If not, a negative result in the experiment could be imputed either to the absence of the effect or to the state of the particles (being in a product state instead of a (anti)symmetrized one). In contrast, if we know by sure that the particles are in a (anti)symmetrized state a negative result could only be interpreted as a demonstration of the absence of the effect.

We propose here a scheme able to verify (anti)symmetrization in two-particle systems, only based on the detection of one of the two particles and the determination of the state of the surviving one. To be concrete, if the state of the two-particle system is (anti)symmetrized the mode distribution of the surviving particle will be a combination of the initial distributions of the two particles. The scheme is, in contrast to previous proposals, a test of identicalness not based on a HOM-type approach.

As an interesting byproduct, the technique also provides an efficient method to generate one-particle superposition states. The surviving particle is in a superposition of the initial states of the two particles. The technique is specially well-suited to generate superpositions of multi-mode states peaked around very different momentum values.

\section{The arrangement}

We consider the same arrangement as that in [12], where the process of detection of one of the particles in a system of two particles in multimode states is analyzed, with special emphasis on the one-particle interferences present in the detection pattern.

Two identical particles are prepared in the state

$$
\left|2_{f g}>=\int d^{3} \mathbf{p} \int d^{3} \mathbf{q} f(\mathbf{p}) g(\mathbf{q}) \hat{a}_{\mathbf{p}}^{+} \hat{a}_{\mathbf{q}}^{+}\right| 0>
$$

where $f$ and $g$ are the mode distributions, which we assume to be normalized, $\int d^{3} \mathbf{p}|f(p)|^{2}=1=\int d^{3} \mathbf{p}|g(p)|^{2}$. In Eq. (1) $\hat{a}_{\mathbf{p}}^{+}$is the creation operator of the 
mode $\mathbf{p}$ and $\mid 0>$ is the vacuum state. The creation and annihilation operators obey the usual relations $\left[\hat{a}_{\mathbf{p}}, \hat{a}_{\mathbf{q}}^{+}\right]_{\mp}=\delta^{3}(\mathbf{p}-\mathbf{q})$. In the double sign expressions the upper one refers to bosons and the lower one to fermions. Note that the above state is not normalized. We shall work with a non-normalized state (see in [12] how this state is normalized), postponing the normalization to the final stage of the calculation.

In all the above expressions we have not included the spin indexes. In order to simplify the presentation we assume the two particles to be in the same spin state making superfluous the presence of the indexes. In terms of the quantum description this assumption is equivalent to have for the spin part of the state the form $\left|s>_{1}\right| s>_{2}$, that is, a product state with 1 and 2 labeling the two particles. We could also have (for both bosons and fermions) a symmetric state for the spin variables, $\left(\left|s>_{1}\right| s^{\prime}>_{2}+\left|s^{\prime}>_{1}\right| s>_{2}\right) / \sqrt{2}$. With any of the two above choices the part of the state related to the spatial variables must be symmetric for bosons and antisymmetric for fermions. We do our presentation in this framework, symmetrized states for the spatial or momentum wave functions of bosons, and antisymmetrized for fermions. The extension to the case of particles in antisymmetrized spin states is straightforward, as it is only necessary to consider antisymmetric and symmetric spatial states for, respectively, bosons and fermions.

The two-particle system now interacts with a detector. We only consider the cases in which only one detection occurs. Moreover, we assume that in the detection process the particle is removed, for instance, by absorption. In order to postselect these cases one must use a detector able to distinguish events with one- or two-detection events (see $[13,14]$ for an optical detector with this property).

Next, we show that the detection process is described by the action of the Schrödinger field operator. From a mathematical point of view this operator $\hat{\psi}(\mathbf{R}, t)$ represents the annihilation of a particle at point $\mathbf{R}$ and time $t[15$, 16]. If the initial state of the system is a n-particle state $|n\rangle$, the action of the operator leaves a $n-1$ particle one, $\hat{\psi}|n>\rightarrow| n-1>$. Thus, this operator represents in a natural way processes in which particles are removed. Intuitively, one can identify this removal process with the destructive detection assumed above. With this identification $\hat{\psi}(\mathbf{R}, t) \mid 2_{f g}>$ describes the state of the resultant one-particle system. At this point a criticism to this identification could be made. Being the detection process a non-unitary one, perhaps we should use a mixture instead of a pure state to describe the final one-particle system. We argue here favoring the pure state choice. Our argument is based on the analogy to detection through photomultipliers in quantum optics [4] (see also [17] for a detailed treatment of absorption in multiparticle massive beams and its description in the framework of the Glauber scheme). In this type of destructive detection the photons are absorbed by the atoms composing the photomultiplier. The description of the interaction between detection device and 
radiation is done in terms of the positive frequency part of the electromagnetic field operator $\hat{\mathbf{E}}^{+}$. If we denote by $\mid R>$ the initial state of the radiation field, its final state is assumed to be $\hat{\mathbf{E}}^{+} \mid R>$, a pure state not a mixture. From that pure state one can evaluate the probability of transition to a state $\left|R_{F}\right\rangle$, as $\left|<R_{F}\right| \hat{\mathbf{E}}^{+}|R>|^{2}$. By analogy with the extensively verified scheme of detection by photomultipliers we assume that after the destructive detection we have the pure state $\hat{\psi}(\mathbf{R}, t) \mid 2_{f g}>$. As we shall see in the following sections, this assumption gives rise to some conclusions that can be experimentally tested, making the assumption verifiable.

In the expression of the field operator $\mathbf{R}$ refers to the point of detection. We use this notation to emphasize that we are considering the detector to be at a fixed position in each repetition of the experiment. We want also remark that it does not act as a physical variable (as it is the case of $\mathbf{r}$ ) but as a parameter. This is a necessary consistency condition for the calculation, because in the second quantization formalism there cannot be explicit dependence on the spatial variables, unless it is in a parametric form [15].

The field operator is given by $\hat{\psi}(\mathbf{R}, t)=\int d^{3} \mathbf{p} \psi_{\mathbf{p}}(\mathbf{R}) \hat{a}_{\mathbf{p}}(t)$, with $\psi_{\mathbf{p}}$ a complete set of orthonormal stationary wavefunctions. The most common choice for this set is that of plane waves $\psi_{\mathbf{p}}(\mathbf{R})=(2 \pi \hbar)^{-3 / 2} \exp (i \mathbf{p} \cdot \mathbf{R} / \hbar)$. The time dependence is carried by the annihilation operator. For planes waves it is given by $\hat{a}_{\mathbf{p}}(t)=\hat{a}_{\mathbf{p}} \exp \left(-i E_{\mathbf{p}} t / \hbar\right)$ with $\hat{a}_{\mathbf{p}}$ the annihilation operator at time $t=0$ and $E_{\mathbf{p}}=\mathbf{p}^{2} / 2 m$ the energy.

From now on, by the matter of simplicity, we shall restrict our considerations to stationary situations. Consequently, we can drop the time variables from all the expressions.

\section{Mode distribution of the surviving particle}

In our case, the final state after one detection is

$$
\hat{\psi}(\mathbf{R}) \mid 2_{f g}>
$$

The evaluation of the state of the surviving particle is simple

$$
\begin{array}{r}
\hat{\psi}(\mathbf{R})\left|2_{f g}>=\int d^{3} \mathbf{p}\left(\psi_{f}(\mathbf{R}) g(\mathbf{p}) \pm \psi_{g}(\mathbf{R}) f(\mathbf{p})\right) \hat{a}_{\mathbf{p}}^{+}\right| 0> \\
=\psi_{f}(\mathbf{R})\left|1_{g}> \pm \psi_{g}(\mathbf{R})\right| 1_{f}>
\end{array}
$$

with $\psi_{f}(\mathbf{R})=\int d^{3} \mathbf{p} f(\mathbf{p}) \psi_{\mathbf{p}}(\mathbf{R})$ and $\left|1_{f}>=\int d^{3} \mathbf{p} f(\mathbf{p}) \hat{a}_{\mathbf{p}}^{+}\right| 0>$.

The above state has not yet been normalized. We denote by $\mid 1_{h}>$ the normalized state of the surviving particle, which is given by $\left|1_{h}>=\hat{\psi}(\mathbf{R})\right| 2_{f g}>$ $/ N$, with

$$
N^{2}(\mathbf{R})=\left|\psi_{f}(\mathbf{R})\right|^{2}+\left|\psi_{g}(\mathbf{R})\right|^{2} \pm 2 \operatorname{Re}\left(\psi_{f}^{*}(\mathbf{R}) \psi_{g}(\mathbf{R})<1_{g} \mid 1_{f}>\right)
$$


Finally, the mode distribution of the surviving particle is

$$
h(\mathbf{p})=\frac{\psi_{f}(\mathbf{R})}{N(\mathbf{R})} g(\mathbf{p}) \pm \frac{\psi_{g}(\mathbf{R})}{N(\mathbf{R})} f(\mathbf{p})
$$

The mode distribution is neither $f$ nor $g$, but a combination of them. The coefficients in the combination are given by some functions depending on the values of the initial wavefunctions at the point of detection.

Note that only in the cases where $\psi_{f}(\mathbf{R})=0$ or $\psi_{g}(\mathbf{R})=0$, the surviving particle has a mode distribution similar to one of the initial ones $\mid 1_{g}>$ or $\left|1_{f}\right\rangle$. This behaviour can be easily understood. Let us consider one of these nodal points, for instance, $\psi_{g}(\mathbf{R})=0$. Then we know by sure that the particle detected is of the type $f$ and, consequently, that the surviving one is of type $g$ $(h(\mathbf{p})=g(\mathbf{p}))$.

In the points where simultaneously we have $\psi_{f}(\mathbf{R})=0$ and $\psi_{g}(\mathbf{R})=0$ the expression for $\left|1_{h}\right\rangle$ is undefined, reflecting the property that at these points the probability of detection of the particles is null and it does not make sense to speak about the surviving one.

The fact that the mode distribution of the surviving particle becomes a combination of the initial ones is a clear manifestation of a superposition process. This follows directly from the last term in Eq. (3), but can easily be visualized if we consider the first-quantization version of the problem, where the wavefunction is given by

$$
\psi_{h}(\mathbf{r})=\int d^{3} \mathbf{p} h(\mathbf{p}) \exp (i \mathbf{p} \cdot \mathbf{r} / \hbar)=\frac{\psi_{f}(\mathbf{R})}{N(\mathbf{R})} \psi_{g}(\mathbf{r}) \pm \frac{\psi_{g}(\mathbf{R})}{N(\mathbf{R})} \psi_{f}(\mathbf{r})
$$

showing the superposition of $\psi_{f}$ and $\psi_{g}$. The wavefunction of the surviving particle is a superposition of the two initial wavefunctions. This superposition can be physically understood in terms of the indistinguishability of the alternatives available to the system, such as stated by the general criterion for quantum superpositions. This criterion states that when the evolution of a quantum system is compatible with several indistinguishable alternatives the state representing the system is a superposition of them. In our case, outside the nodal points, the detector is unable to provide information about the mode distribution of the particle measured, making the alternatives detected particle with mode distribution $f$ and detected particle with mode distribution $g$ indistinguishable.

When the particles are not in (anti)symmetrized states the above results do not hold. In the second-quantization formalism used in this paper the absence of (anti)symmetrization translates into the use of (anti)commuting creation and annihilation operators $\hat{a}$ and $\hat{b}$. The initial state (now normalized) is

$$
\left|2_{f g}>_{N S}=\int d^{3} \mathbf{p} \int d^{3} \mathbf{q} f(\mathbf{p}) g(\mathbf{q}) \hat{a}_{\mathbf{p}}^{+} \hat{b}_{\mathbf{q}}^{+}\right| 0>
$$


If we place a detector of particles of type $a$ (the reasoning for type $b$ is similar) the state after one-detection event is

$$
\hat{\psi}^{(a)}(\mathbf{R})\left|2_{f g}>_{N S}=\psi_{f}^{(a)}(\mathbf{R})\right| 1_{g}>^{(b)}
$$

The above expression shows that now the mode distribution of the surviving particle is identical to the initial one.

\section{The scheme}

The above results provide the basis for a scheme of (anti)symmetrization verification between the two initial particles. The scheme has two steps. In the first stage, the two particles interact with the detector. We postselect all the cases of one-detection events. Detectors able to distinguish one- and two-detection events would greatly simplify the task. As signaled before, in the optical case such types of detectors are available $[13,14]$. In the second step, we deal with the postselected surviving particles. We must test if the particles are in one of the two initial states or in a superposition of them. To this end we can use standard tomography techniques or study the spatial detection patterns of the surviving particles. If the detectors used in the first step are unable to discriminate between one- and two-detection events the postselection must also be carried out in the second stage. Then we choose the cases in which, in each repetition of the process, there is an event in the detector and another in the device testing the superposition. As the efficiency of the detectors of massive particles is in general high the scheme seems to be, in principle, an effective one. These are the general lines of the scheme. Now we discuss in detail a particular realization that is, in principle, viable with present technology. We present it in Fig. 1.

We first consider the stage of preparation of the state. We consider an arrangement where each particle is emitted by a different source. Two main types of sources have been used in the study of (anti)bunching effects in massive particles, magnetic traps $[18,19]$ and optical lattices $[20,21]$. Sometimes the traps and lattices use previously generated Bose-Einstein condensates (BEC), and in other occasions gases produced by evaporative and sympathetic cooling. In $[19,20,21]$ the atoms are released at the switch-off of the potentials retaining the atoms in the trap or lattice. A number of atoms of the order of $10^{4}$ to $10^{5}$ are released, making the technique useless for our purpose of preparing twoparticle systems. From our point of view, more interesting is the method of [18], where a weak continuous microwave field is applied to the BEC in the magnetic trap for output coupling an atom laser. Some atoms inside the trap are spin-flipped and do not longer experience the magnetic potential, leaving the trap. The number of released atoms is much smaller than in the previous techniques. As a matter of fact, in [18] a mean atom number very close to 2 was reported, making possible the individual detection of the atoms. With this 
method, adequate values of the microwave fields, and synchronization of the two fields acting on different traps it seems possible to create two-particle states.

For different parameters of the two traps (different cooling,...) we can have particles with different momentum distributions. Another way to generate different momentum distributions is to collimate the released beam via delimiters of different apertures. The uncertainty relation tell us that if the width of the aperture is $l$ the momentum spread will be $\delta p \sim \hbar / l$.

When the mean separation between the emission of successive pairs of particles is large enough the postselection process of pairs can be done in a simple way by comparing the detection times in the detector and the tomographic device.
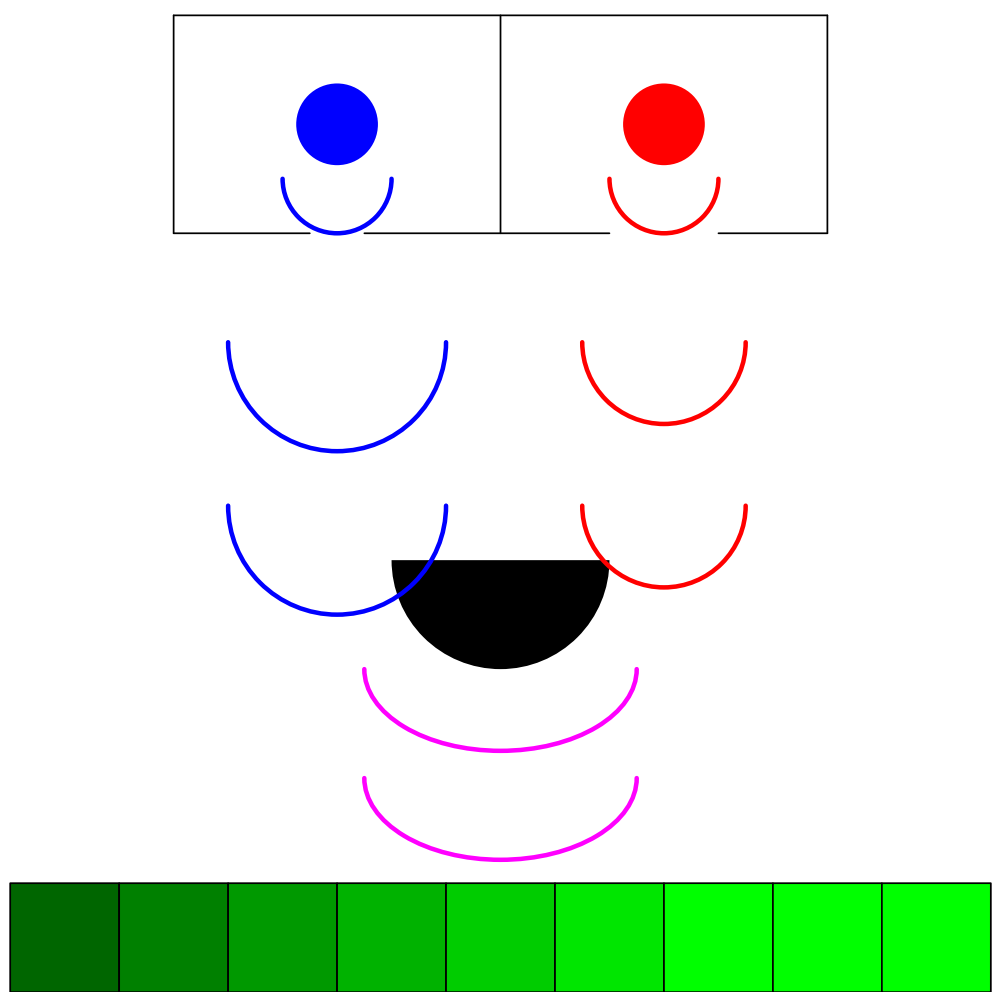

Figure 1: Schematic representation of the proposed arrangement. The boxes containing the red and blue circles represent the two sources of particles, with typical mode distributions $f$ and $g$. The black half-circle corresponds to the main detector. Finally, the green boxes in the bottom of the figure represent the detectors array.

Next, we must perform tomography of the surviving particle in order to 
determine its state. As it is well-known [22], the first question in quantum tomography is to determine if the system is in a pure state or it must be described by a mixture. In the first case, measuring the detection probability in different planes along the beam propagation direction we can determine the complete (modulus and phase) wave function [22]. In the second case, only the density matrix of the system can be obtained from the measurements. Then our first task is to determine if we are dealing with a pure state or a mixture. In Sect. 2 we have assumed that after the detection the two-particle system collapses into a pure one-particle state. A possible way to assess if the surviving particle is in a pure superposition state consists in the comparison of the spatial detection patterns of the surviving and separately emitted particles. To this end we place a detectors array downstream of the main detector (that absorbing one of the particles). With this array we can determine the spatial detection pattern. For the surviving particle in a pure state we have the distribution

$$
\begin{array}{r}
\left|\psi_{h}\left(\mathbf{r}_{\text {det }}\right)\right|^{2}=\left|\alpha_{f}(\mathbf{R})\right|^{2}\left|\psi_{g}\left(\mathbf{r}_{\text {det }}\right)\right|^{2}+\left|\alpha_{g}(\mathbf{R})\right|^{2}\left|\psi_{f}\left(\mathbf{r}_{\text {det }}\right)\right|^{2} \\
\pm 2 \operatorname{Re}\left(\alpha_{f}^{*}(\mathbf{R}) \psi_{g}^{*}\left(\mathbf{r}_{\text {det }}\right) \alpha_{g}(\mathbf{R}) \psi_{f}\left(\mathbf{r}_{\text {det }}\right)\right)
\end{array}
$$

with an obvious notation for the $\alpha$ coefficients, and $\mathbf{r}_{\text {det }}$ denoting the location of the detectors composing the array.

In a different run of experiments we obtain $\left|\psi_{f}\left(\mathbf{r}_{d e t}\right)\right|^{2},\left|\psi_{g}\left(\mathbf{r}_{d e t}\right)\right|^{2},\left|\psi_{f}(\mathbf{R})\right|^{2}$ and $\left|\psi_{g}(\mathbf{R})\right|^{2}$ by considering situations where only one of the sources emits particles. Then we compare $\left|\psi_{h}\left(\mathbf{r}_{\text {det }}\right)\right|^{2}$ with $\left|\psi_{f}(\mathbf{R})\right|^{2}\left|\psi_{g}\left(\mathbf{r}_{\text {det }}\right)\right|^{2}+\left|\psi_{g}(\mathbf{R})\right|^{2}\left|\psi_{f}\left(\mathbf{r}_{\text {det }}\right)\right|^{2}$ in order to see if both distributions coincide (there is no superposition of the initial states in the surviving particle, corresponding the situation to a mixture) or not (there is a superposition associated with a pure state). Note that we do not need to determine the normalization factor $N(\mathbf{R})$ because it is not necessary to see if both distributions have the same analytical form.

With the above procedure we can determine the presence of the superposition in the surviving particle and, consequently, if it is in a pure state. If it is so, we can complete the tomographic process by position measurements of the particle in different planes after the main detector (a detectors array at each one of these planes).

It must be noted that if we only want to know if the two-particle state was (anti)symmetrized previous to the interaction with the main detector, it is enough to carry out the above procedure of comparing the $\left|\psi_{h}\right|^{2}$ and $\left|\psi_{f}\right|^{2}$ and $\left|\psi_{g}\right|^{2}$ distributions, without need of a much more demanding complete process of tomography. In effect, when the surviving particle is in a superposition state the initial two-particle wave function necessarily had to be in a (anti)symmetric state. The situation is different if, in addition to determine the presence of exchange processes in the two-particle system previous to the interaction with the main detector, we want to know if the spatial part of the state of the system was in a symmetric or antisymmetric state. As discussed before, the bosonic and fermionic particles can be in a symmetric or antisymmetric spatial state 
depending on the antisymmetric or symmetric character of the spin part of the state. The symmetric or antisymmetric character of the spatial part of the two-particle wave function can be determined from the positive or negative sign in the superposition of the surviving particle. However, the value of this sign cannot be determined with the method of comparison of $\left|\psi_{h}\right|^{2}$ and $\left|\psi_{f}\right|^{2}$ and $\left|\psi_{g}\right|^{2}$ presented in the previous paragraphs. We would need to know the complete expression of $\psi_{h}$ (or equivalently, the knowledge of $\psi_{f}$ and $\psi_{g}$ and the use of Eq. (9)). We would need a complete tomography process.

\section{Conclusions}

We have presented in this paper a scheme for (anti)symmetrization verification. Our proposal is not based on HOM-type arrangements. The ideas presented here seem, in principle, accessible to experimental scrutiny.

The scheme could also be used to generate superposition states in oneparticle systems, starting from two-particle ones. If the initial two-particle state is (anti)symmetrized, the detection of one of the members of the pair outside the nodal points leaves the other in a superposition of the two initial states. We have generated superposition via (anti)symmetrization and detection. An interesting application of the method would be to generate a multi-mode superposition state of two very different central momenta. We only need (i) to prepare the initial particles with momentum distributions $f(p)$ and $g(p)$ peaked around two values $p_{f}$ and $p_{g}$ with $\left|p_{f}-p_{g}\right| \gg \sigma$, where $\sigma$ is the typical width of the distributions, (ii) to get a non-negligible overlapping of the two particles in order to have a (anti)symmetrized state, and (iii) to postselect the cases with one-detection events.

Finally, we must consider the possibility of applying the scheme in quantum optics. The HOM technique with beam splitters is very efficient for optical systems, making superfluous the existence of other verification schemes. Nevertheless, our proposal could be interesting to generate superpositions of one-photon states. A simple calculation shows that a result similar to that obtained for the distribution of the surviving massive particle holds in quantum optics. Moreover, in this field there is much experience in dealing with two-photon states, even in multimode states [4].

\section{References}

[1] van Enk, S. J., Lütkenhaus, N. and Kimble H. J., Phys. Rev. A 75, 052318 (2007).

[2] Plenio, M. B. and Virmani, S., Quant. Infor. Comput. 7, 1 (2007).

[3] Hong, C. K., Ou, Z. and Mandel, L., Phys. Rev. Lett. 592044 (1987). 
[4] Loudon, R., "The Quantum Theory of Light" (Oxford Science Publications, Oxford, 2000).

[5] Liu, R. C., Odom, B., Yamamoto, Y. and Tarucha, S., Nature 391, 263 (1998).

[6] Oliver, W. D., Kim, J., Liu, R. C. and Yamamoto, Y., Science 284, 299 (1999).

[7] Bose, S. and Home, D., Phys. Rev. Lett. 88, 050401 (2002).

[8] Lim, Y. L. and Beige, A., New J. Phys. 7, 155 (2005).

[9] Adams, C. S., Sigel, M. and Mlynek, J., Phys. Rep. 240143 (1994).

[10] Croning, A. D., Schmiedmayer, J. and Pritchard, D. E., Rev. Mod. Phys. 81, 1051 (2009).

[11] Sancho, P., J. Phys. B 43, 065504 (2010); ibid, Phys. Rev. A 82033814 (2010).

[12] Sancho, P., J. Phys. A 37, 11003 (2004).

[13] Golovin, V. and Saveliev, V., Nucl. Instr. Meth. Phys. Res. 518, 560 (2004).

[14] Eraerds, P., Legré, M., Rochas, A., Zbinden, H. and Gisin, N., Opt. Exp. 15, 14539 (2007).

[15] Landau, L. D. and Lifshitz, E. M., "Quantum Mechanics" (Addison-Wesley, 1965).

[16] Baym, G., "Lectures on Quantum Mechanics" (W. A. Benjamin, 1969).

[17] Sancho, P., Ann. Phys. 323, 1271 (2008).

[18] Öttl, A., Ritter, S., Köhl, M. and Esslinger, T., Phys. Rev. Lett. 95, 090404 (2005).

[19] Jeltes, T., McNamara, J. M., et al, Nature 445, 402 (2007).

[20] Fölling, S., Gerbier, F., Widera, A., Mandel, O., Gericke, T. and Bloch, I., Nature 434, 481 (2005).

[21] Rom, T., Best, Th., van Oosten, D., Schneider, U., Fölling, S., Paredes, B. and Bloch, I., Nature 444, 733 (2006).

[22] Lvovsky, A. I. and Raymer, M. G., Rev. Mod. Phys. 81, 299 (2009). 